Case Report

\title{
Intraprocedural Stent Thrombosis: Case Series of a Rare Complication Managed Successfully
}

\author{
Nikesh Jain ${ }^{1, *}$, Nilesh Tawade ${ }^{2}$, Nihar Mehta ${ }^{1}$, Ajit Desai ${ }^{1}$, Ashwin Mehta ${ }^{1}$ \\ ${ }^{1}$ Jaslok Hospital and Research Centre, Mumbai, India \\ ${ }^{2}$ Narayana Institute of Cardiac Sciences, Bangalore, India \\ Email address: \\ drnikeshjain@gmail.com (N. Jain) \\ ${ }^{*}$ Corresponding author
}

\section{To cite this article:}

Nikesh Jain, Nilesh Tawade, Nihar Mehta, Ajit Desai, Ashwin Mehta. Intraprocedural Stent Thrombosis: Case Series of a Rare Complication Managed Successfully. Cardiology and Cardiovascular Research. Vol. 3, No. 2, 2019, pp. 31-36. doi: 10.11648/j.ccr.20190302.13

Received: May 1, 2019; Accepted: June 10, 2019; Published: June 26, 2019

\begin{abstract}
Intra-procedural stent thrombosis (IPST) is defined as the development of occlusive or non-occlusive new thrombus in or adjacent to a recently implanted stent before the PCI procedure is completed. The frequency of occurrence currently ranges between $0.5-1.7 \%$ of all PCI procedures and it seems to be considerably reduced with newer drug eluting stents and improved techniques. The occurrence of IPST is relatively rare, even in ACS patients, and is related strongly to clinical presentation and procedural factors (e.g., anticoagulation regimen, lesion type, presence of thrombus at baseline, stent under expansion and edge dissection etc.) than to baseline demographic characteristics. Imaging modalities like IVUS or OCT can prevent as well as identify the underlying cause for IPST. IPST not only decreases success rate of PCI but is also associated with higher rates of slow flow or no reflow, distal embolization and side branch closure. It is also associated with higher mortality, myocardial infarction, ischemia driven revascularisation and stent thrombosis on follow up. IPST can be managed immediately in cath lab and involves use of GP2b3a inhibitors, optimising stent apposition by repeat balloon dilatation, use of imaging to identify the cause and accordingly corrective measures to be taken. Rarely emergency CABG may be required if underlying cause cannot be corrected. Prevention is the key. IPST can be prevented by using newer anti-platelets, use of GP2b3a inhibitors especially in presence of ACS or high thrombus load, preparing the lesion well before stenting, use of atherectomy devices when needed, appropriate stent size selection and use of imaging modalities in complex lesions to optimise stent selection and apposition. We describe three cases of the intra procedural stent thrombosis under different clinical scenarios and its management.
\end{abstract}

Keywords: Intraprocedural Stent Thrombosis, Intravascular Imaging, Newer Anti Platelets

\section{Introduction}

Stent thrombosis (ST) after percutaneous coronary intervention $(\mathrm{PCI})$ is an uncommon but serious complication. A standard definition of stent thrombosis was proposed by an Academic Research Consortium (ARC) in order to make it possible to compare the true rates of stent thrombosis across different trials and registries. The definition categorises stent thrombosis according to the level of documentation and timing as definite or probable or possible; or based on the elapsed time since stent implantation as early or late or very late. But Intra-procedural stent thrombosis (IPST) is excluded from standard ARC definition of stent thrombosis.

Intra-procedural stent thrombosis (IPST) is defined as the development of occlusive or non-occlusive new thrombus in or adjacent to a recently implanted stent before the PCI procedure is completed.

The frequency of occurrence currently ranges between 0.5 $-1.7 \%$ of all PCI procedures. IPST occurred in $0.7 \%$ of PCI when frame-by-frame analysis was done for 6591 patients enrolled in the ACUITY and HORIZONS-AMI (Harmonizing Outcomes With Revascularization And Stents 
In Acute Myocardial Infarction) trials [1]. IPST not only decreases success rate of PCI but is also associated with higher rates of slow flow or no reflow, distal embolization and side branch closure. It is also associated with higher 1 -year mortality and morbidity at one year post-PCI. One year follow-up data shows a $41.1 \%$ rate of death, MI, or TVR in patients who had experienced IPST versus $14.5 \%$ in patients with no IPST [1]. Other adverse events were also increased in patients with IPST after one year post-PCI, such as post procedural stent thrombosis, and non-CABG major bleeding.

\section{Case Series}

This case series describes three challenging cases of intraprocedural thrombosis during PCI.

\subsection{CASE 1}

A 57 year old diabetic and hypertensive lady with history of PCI to the LAD with $3.0 \times 16 \mathrm{~mm}$ Paclitaxel eluting stent (PES), had progressive Angina on exertion class III since 6 months with recent onset of rest angina. Her ECG had new anterolateral ischemic changes with $2 \mathrm{D}$ echocardiography showed preserved ejection fraction. She was loaded $300 \mathrm{mg}$ of Aspirin and 600mg of Clopidogrel.

Coronary Angiography revealed an $80 \%$ instent restenosis in the $\mathrm{LAD}$ OM showed $70 \%$ lesion in the proximal segment.

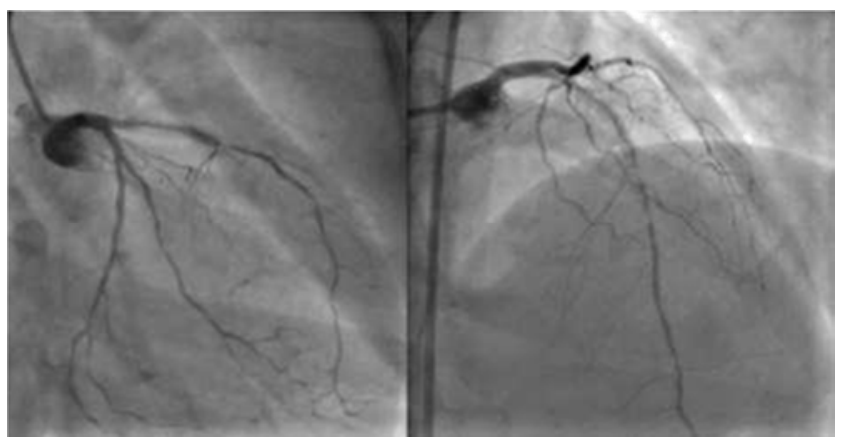

Figure 1. Instent restenosis of the LAD followed by critical stenosis.

The LAD lesion was crossed with a hydrophilic wire and the distal lesion was dilated with a 2 X $20 \mathrm{~mm}$ Semi-compliant (SC) balloon upto 20 atmospheres (atm) but it did not yield (figure 2 A). A $2.25 \times 10 \mathrm{~mm}$ Cutting balloon was used at 14 atm without success. Rotational atherectomy was then performed with a $1.25 \mathrm{~mm}$ Rotaburr was used at 180,000 rpm for 16 seconds. However, the lesion did not yield even after rotablation. Therefore, the lesion was dilated with $2.25 \times 10$ mm Non-Complaint (NC) balloon at 24 atm The lesion seemed to have been adequately modified. IVUS was attempted but the catheter could not cross the lesion. The lesion was then stented with $2.25 \times 30 \mathrm{~mm}$ Zotarolimus eluting stent (ZES) at 16 atm for 20 seconds. The proximal part of the stent was underexpanded hence postdilated with $2.5 \times 10 \mathrm{NC}$ balloon at $20 \mathrm{~atm}$. The stent did not expand furthur. Since there was TIMI III flow in the LAD the procedure was terminated. (figure $2 \mathrm{C}$ ).

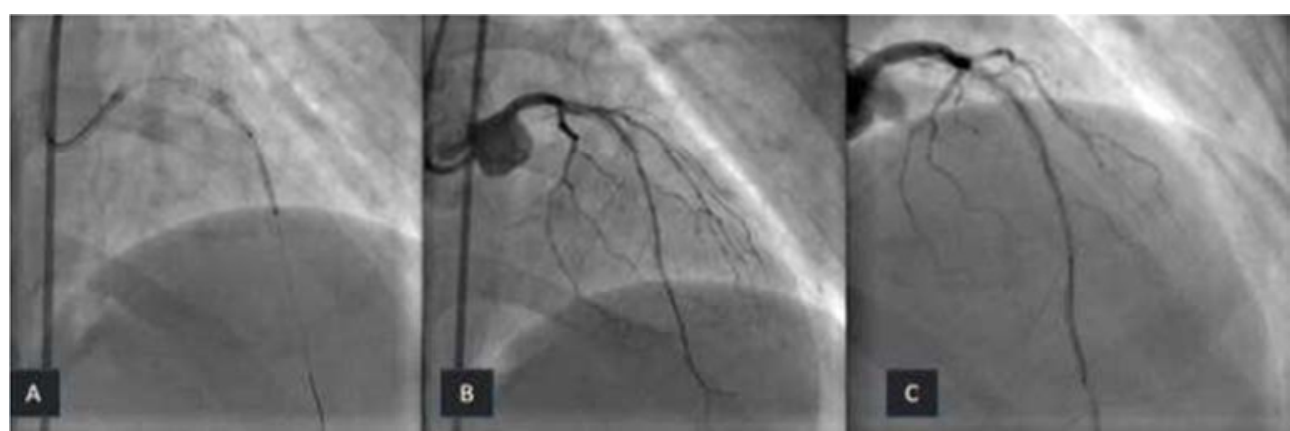

Figure 2. (A) Non-dilatable lesion in the LAD segment. (B) After balloon dilatation. (C) Lesion was then treated with rotablation.

In the recovery room, the patient complained of severe chest pain within few minutes and the ECG showed ST elevation in I, aVL. Check angiography showed acute stent thrombosis in LAD stent with TIMI I flow (Figure 3 A).

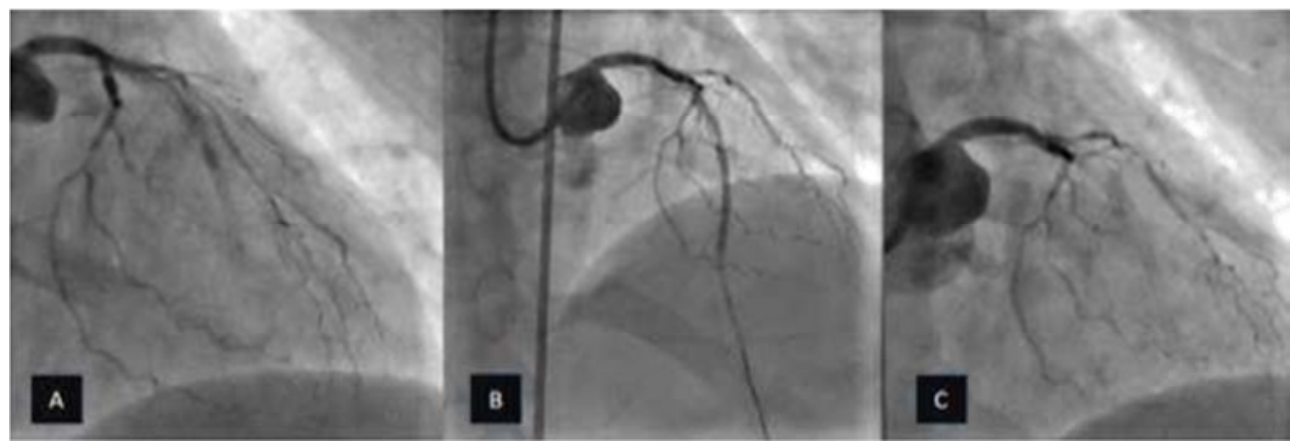

Figure 3. (A) Depicts intraprocedural stent thrombosis which was treated with serial balloon dilatation resulted in TIMI III flow (B) but there was recurrence of thrombosis (C). 
The lesion was dilated with 2.5 and $2.75 \mathrm{~mm}$ NC balloons serially. Abciximab infusion was started. TIMI III flow returned to the LAD and the patient was shifted to recovery room. However within half an hour there was recurrence of chest pain with ST elevation in I and aVL. Check angiography revealed complete occlusion of LAD stent with thrombus. Several runs of thrombosuction were was done followed by serial inflation of the proximally stented LAD. TIMI III flow in the LAD was re-established. The patient, however, developed haemodynamic instability with several runs of Ventricular tachycardia. In view of repeated intra procedural stent thrombosis (IPST), the patient underwent successful emergency $\mathrm{CABG}$ surgery with left internal mammary artery graft to LAD.

\subsection{CASE 2}

A 64-year-old diabetic and hypertensive lady presented progressive dyspnoea on exertion since 3 years, positive stress test and a normal LV ejection fraction. She was given with
$300 \mathrm{mg}$ of Aspirin and $60 \mathrm{mg}$ of Prasugrel. Her Coronary Angiography revealed calcified coronary vessels, 60-70\% borderline lesion in mid LAD. Fractional flow reserve (FFR) showed that the lesion was functionally significant. In view of severe calcification, rotational atherectomy was done with a $1.5 \mathrm{~mm}$ Rotaburr at 1,70,000 rpm. Then the lesion was dilated with $2.5 \times 15 \mathrm{~mm}$ SC balloon and subsequently stented with $2.5 \times 22 \mathrm{~mm}$ Zotarolimus eluting stent (ZES) stent at $10 \mathrm{~atm}$.

Immediately after procedure, the patient developed chest heaviness with ST elevation in aVL. Check shoot showed there was a spasm of LAD distal to stent, which was treated with intra coronary NTG boluses. The stent was dilated with a $2.5 \times 12 \mathrm{~mm}$ balloon. However, the chest pain and ST elevation in aVL persisted.

The subsequent angiography shoot showed haziness within the stent and proximal to stent (figure 4) thought to be a dissection. It was stented with $2.5 \times 14 \mathrm{~mm}$ ZES stent. The ST elevation returned to baseline. Check shoot showed excellent result and patient was observed closely in recovery room.

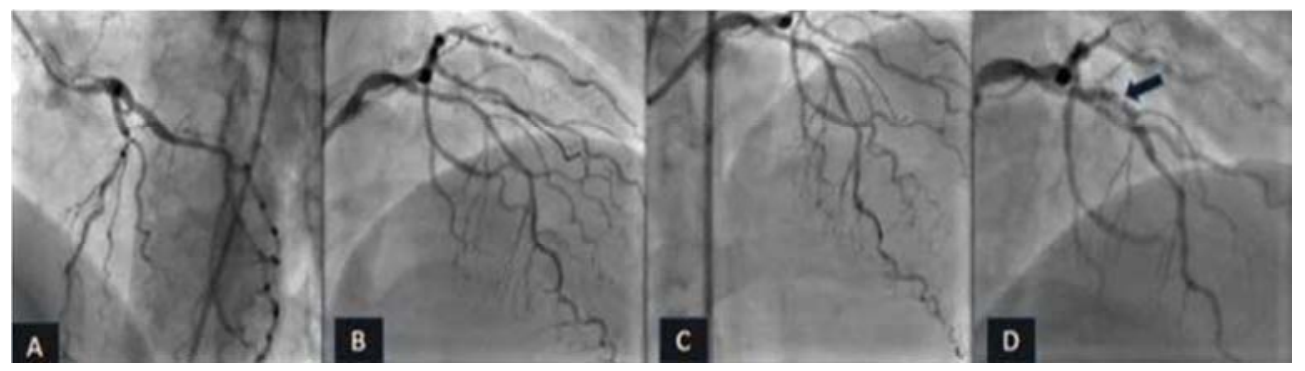

Figure 4. (A) LAO cranial view showing significant stenosis in mid LAD. (B) (C) there was spasm in mid LAD post and diagonal branches rotablation. (D) there was immediate Intra procedural stent thrombosis after stenting.

Patient developed angina again in few minutes, with ST elevation in avL. Angiography revealed intra stent filling defects and haziness suggestive of the acute stent thrombosis and a diagnosis of IPST (Intra Procedural Stent Thrombosis) was made (figure 4D).

The patient was given intra-coronary Abciximab followed by an infusion. Balloon dilatation was done with a $2.75 \mathrm{x}$ $10 \mathrm{~mm}$ NC balloon upto 16 atm. Check shoot showed good result with TIMI III flow.

After 30 minutes, the patients check angiography shoot showed recurrent stent thrombosis, for which dilatation was done with a 3 X $12 \mathrm{NC}$ balloon upto $18 \mathrm{~atm}$. (figure $5 \mathrm{~A}$ ).

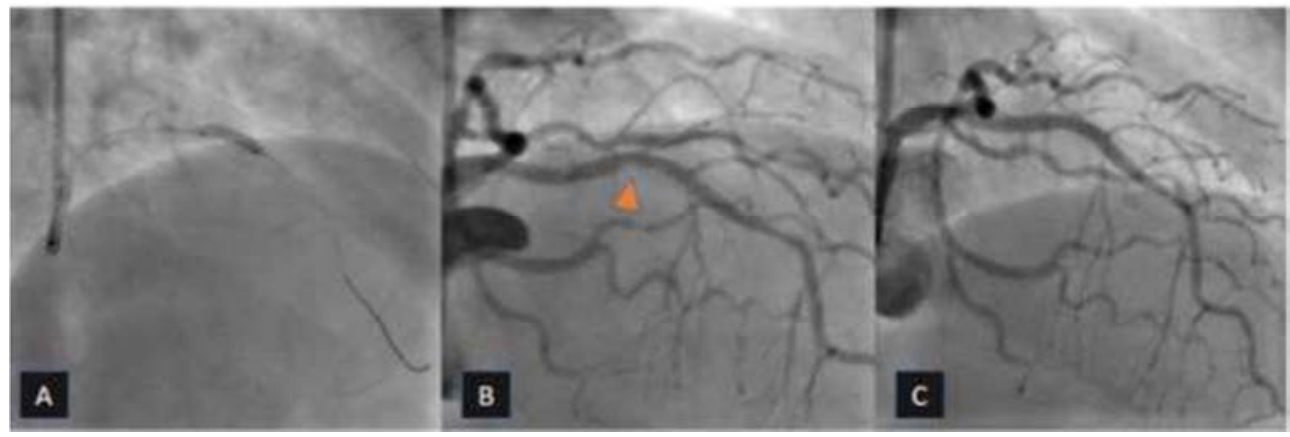

Figure 5. (A) multiple balloon dilatations were done and Abciximab infusion was started. (B) Post balloon dilatation residual thrombus (arrow head) (C) Check angiography at the end of procedure showed a good final result with TIMI III flow.

The angiography showed a good final result with TIMI III flow. She was shifted to the ICU after 30 minutes on abciximab and heparin infusion. She was shifted from Prasugrel to Ticagrelor. The remaining stay of the patient in hospital was uneventful.

\subsection{CASE 3}

A 78-year-old diabetic and hypertensive lady with history of dyspnoea on exertion NYHA class II since 10 days and recent onset rest angina had diffuse $\mathrm{T}$ inversion on 
electrocardiogram and a normal LV ejection fraction on echocardiography. Patient was loaded with 300mg of Aspirin and $450 \mathrm{mg}$ of Clopidogrel.

Her coronary angiography showed significant lesions in the LAD, diagonal and mid circumflex artery. The proximal and mid right coronary artery also showed diffuse lesions of $80 \%$ severity extending upto the distal right coronary artery. The patient refused CABG surgery.

The RCA was addressed first and the lesions were dilated with a $2.0 \times 12 \mathrm{~mm}$ SC balloon at nominal pressures followed by stenting with a 2.25 X $30 \mathrm{~mm}$ ZES in the mid RCA.

Check shoot suggestive of no flow from mid RCA with dissection at mid segment.

The midsegment was stented with another 2.25 x $30 \mathrm{~mm}$ ZES overlapping the distal RCA. Check shoot showed TIMI I flow with haziness within the distal stent and filling defects. The patient developed ST elevation in inferior leads followed by bradycardia with hypotension which was treated with Atropine, IV fluid and ionotropic support (Dopamine $5 \mathrm{mcg}$ $/ \mathrm{kg} / \mathrm{min})$.

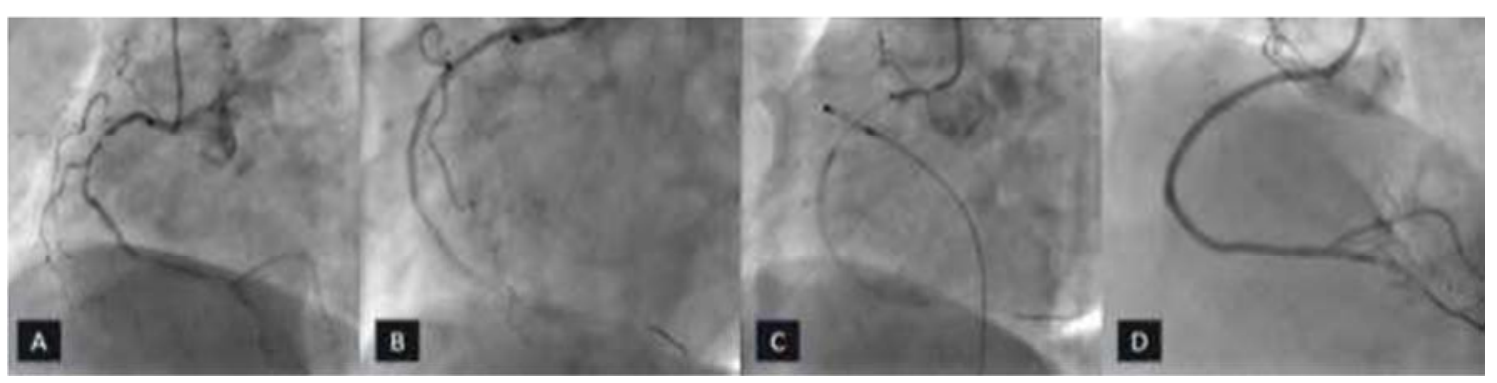

Figure 6. (A) RAO cranial view showing RCA with significant calcified stenosis at proximal and mid segments. (B) (C) post stenting there was no-reflow as well as evidence of thrombus in the stented region (pacemaker was inserted to tackle bradycardia). (D) After balloon dilatation and intracoronary Abciximab injection.

In view of new thrombus within distal stent a diagnosis of IPST (Intra Procedural Stent Thrombosis) made (Figure 6) and patient was given intracoronary Abxicimab bolus followed by an infusion. The stent was dilated with $2 \times 20 \mathrm{~mm}$ $\mathrm{NC}$ balloon upto $14 \mathrm{~atm}$ from distal to proximal segment sequentially.

The proximal RCA was stented with $2.25 \times 28 \mathrm{~mm}$ everolimus eluting stent overlapping with the mid RCA stent at 12 atm.

Check shoot showed TIMI III flow but there was haziness distal to distal RCA stent. That segment was dilated with $2.0 \mathrm{x}$ $15 \mathrm{~mm}$ balloon upto16 atm and subsequently stented with a $2.25 \mathrm{X} 13 \mathrm{~mm}$ sirolimus eluting stent. The stent was post dilated upto 18 atm and the final angiography showed TIMI III flow. The patient was hemodynamically stable and the remaining course in hospital was uneventful.

\section{Discussion}

Chieffo et al. reported that Intraprocedural Stent Thrombosis (IPST), defined as an angiographically intraluminal filling defect within the stent, occurred in $0.7 \%$ of cases during elective implantation of Sirolimus-Eluting Stents (SES) in 670 patients in the absence of acute MI, thrombus containing lesions, and residual persistent dissections [2].

In the current era, stent thrombosis is a rare but potentially catastrophic complication of stent implantation. Stent thrombosis is called "acute" when it occurs within 24 hours of stent placement and "subacute" when it happens within 24 hours to 30 days of intervention [1]. These two groups are often combined and described as "early" stent thrombosis. The term "late" stent thrombosis describes events happening 30 days to 1 year after intervention; stent thrombosis occurring after 1 year is called "very late" [3]. Initial clinical experiences with coronary stents witnessed early stent thrombosis rates of around $10 \%$. The incorporation of routine high-pressure balloon inflations during stent deployment along with the adjunctive use of dual antiplatelet therapy reduced the rate of early stent thrombosis to the current level of less than $1 \%$ with similar rates for bare-metal and drug-eluting stents $[4,5]$.

Table 1. The predictors of IPST, which have been suggested so far in the literature, include Procedural factors and patient related factors include [6].

\begin{tabular}{ll}
\hline Procedural factors & Patient Related Factors \\
\hline Bifurcation stenting & Acute Coronary Syndrome \\
Presence of residual dissection & Diabetes Mellitus \\
Thrombus & Chronic Renal Failure \\
Stent under expansion (most common) & Antiplatelet Drug Resistance \\
Tissue protrusion & Inadequate loading of antiplatelets \\
Long lesion and stent length & \\
Small Vessel Or Stent diameter & \\
Smaller post procedural luminal diameter & \\
Venous graft interventions & \\
Primary PCI & \\
\hline
\end{tabular}


Stent thrombosis typically manifests as an ST-segment elevation acute myocardial infarction. These are very serious events associated with death in $10 \%$ to $15 \%$ of cases at 30 days and myocardial infarction in about $60 \%[3,4]$. Predictors of Intraprocedural instent thrombosis are enumerated in table 1.

The most common abnormality observed on intravascular ultrasound is under expansion of the stent, resulting in a smaller minimal luminal area compared to patients without stent thrombosis. Additionally, residual dissection, thrombus, or tissue protrusion may be evident. Intravascular ultrasound (IVUS) and OCT (Optical Coherence Tomography) identifies mechanisms of stent failure by providing complementary information regarding the extent and distribution of neointima arterial remodelling, stent under expansion, and incomplete stent apposition (ISA) [7, 8].

The three cases described above highlight the different scenarios of the intra procedural stent thrombosis.

In the first case, inadequate burring could have provoked the milieu for the thrombus generation during the procedure. We speculate that under-burring (burr to artery ratio was < 0.50 ) and under-preparation of lesion could have caused the stent under expansion which lead to stent thrombosis. Hence we recommend proper selection of burr size, even in the presence of instent restenosis, to adequately debulk the lesion to facilitate the proper stent expansion. Rotabalation was also used in second case raising the notion that platelet activation and thermal injury could be contributory factors for IPST.

In second and third case, dissection adjacent to the stented segment could have triggered the intraprocedural stent thrombosis. All three patient had diabetes, a well known risk factor for the IPST. Other notable factor in the first and third case could be the resistance to the antiplatelet drugs, notably clopidogrel as it needs to be metabolized to an active form with the activity of a cytochrome P450. Variants of this enzyme due to mutations in the gene CYP2C19, can lead to changes in the metabolism of clopidogrel and its bio-availability [9]. Western data reported incidence of clopidogrel resistance to be $13-19 \%$ but Indians are more prone for poor metabolism of the clopidogrel, resistance to clopidogrel reported upto $28 \%[10,11]$.

The best management strategy for coronary thrombotic lesions is still controversial. Restoration of vessel patency is the major goal of management; most operators begin with balloon angioplasty to achieve this. Thrombus aspiration is a treatment option which can improve the procedural success.

Once reperfusion has been accomplished, evaluation of the stent and adjacent arterial segments with intravascular ultrasound (IVUS) or optical coherence tomography (OCT) may provide valuable insight into the mechanism of stent thrombosis and may directly impact management. Imaging with IVUS or OCT may demonstrate an edge dissection, inadequate stent expansion, inadequate stent apposition, stent fracture, tissue protrusion or early stent thrombosis that can guide the further management. If imaging is not used, it is advisable to perform additional balloon inflations within the stent using either a larger diameter, noncompliant balloon or higher pressure than originally used.

Adjunctive use of glycoprotein IIb/IIIa inhibitors is strongly recommended, particularly because the thrombus causing stent occlusion is rich in platelets. Adjunctive Abciximab therapy in primary angioplasty for high-risk patients is known to be beneficial [12].

"CHAMPION PHOENIX" [13] trial is the largest study to specifically evaluate the incidence, predictors, and impact of IPST on the early prognosis of patients undergoing elective, urgent, and emergent PCI. The main results of the present study are as follows:

IPST occurred in approximately $1 \%$ of patients undergoing PCI;

NSTE-ACS or STEMI at presentation, the presence of angiographic thrombus, and the total stent length implanted were independently associated with the occurrence of IPST,

IPST was associated with a significant increase in mortality, MI, Ischemia driven revascularization, and stent thrombosis at $48 \mathrm{~h}$ and at 30 days; and

Cangrelor use was independently associated with freedom of IPST; treatment with Cangrelor compared to clopidogrel at the time of the PCI procedure significantly decreased the occurrence of IPST and adverse events through 30 day

These three cases highlight the different scenarios of the intra procedural stent thrombosis.

\section{Conclusion}

The occurrence of IPST is relatively rare, even in ACS patients, and is related strongly to clinical presentation and procedural factors (e.g., anticoagulation regimen, lesion type, presence of thrombus at baseline, stent under expansion and edge dissection etc.) than to baseline demographic characteristics. The development of IPST is strongly associated with a substantial morbidity, which implies that these patients might especially benefit from more potent antiplatelet agents, such as prasugrel or ticagrelor. Intra-procedural ST is also a powerful independent predictor of 1-year mortality. Loading of the appropriate antiplatelets is very important step to prevent the IPST. Imaging modality like IVUS and OCT should be used to ensure adequate stent deployment and to detect thrombus/edge dissections to prevent IPST and its disastrous consequences.

\section{References}

[1] S J. Brener, E Cristea, A J. Kirtane., et al. Intra-procedural stent thrombosis: a new risk factor for adverse outcomes in patients undergoing percutaneous coronary intervention for acute coronary syndromes." JACC Cardiovasc Interv 6 (1): 36-43.

[2] A Chieffo, E Bonizzoni, D Orlic et al. Intraprocedural Stent Thrombosis During Implantation of Sirolimus-Eluting Stents. Circulation. 2004; 109: 2732-2736.

[3] Lemesle G, Delhaye C, Bonello L, et al. Stent thrombosis in 2008: Definition, predictors, prognosis and treatment, Arch Cardiovasc Dis 101:769-777, 2008. 
[4] Ong ATL, Hoye A, Aoki J, van Mieghem CAG, et al. Thirty-day incidence and six-month clinical outcome of thrombotic stent occlusion after bare-metal, sirolimus, or paclitaxel stent implantation, J Am Coll Cardiol 45:947-953, 2005.

[5] Cutlip DE, Baim DS, Ho KKL, et al. Stent thrombosis in the modern era: A pooled analysis of multicenter coronary stent clinical trials, Circulation 103:1967-1971, 2001.

[6] Cheneau E, Leborge L, Mintz GS, et al. Predictors of subacute stent thrombosis: Results of a systematic intravascular ultrasound study, Circulation 108:43-47, 2003.

[7] Uren NG, Schwarzacher SP, Metz JA, Lee DP, Honda Y, Yeung AC, Fitzgerald PJ, Yock PG. Predictors and outcomes of stent thrombosis. Eur Heart J. 2002; 23: 124-132.

[8] Fujii K, Carlier SG, Mintz GS, Yang YM, Moussa I, Weisz G, Dangas G, Mehran R, Lansky AJ, Kreps EM, Collins M, Stone GW, Moses JW, Leon MB. Stent underexpansion and residual reference segment stenosis are related to stent thrombosis after sirolimus-eluting stent implantation. J Am Coll Cardiol.2005; 45: 995-998.
[9] Jose R, Chandrasekaran A, Sam SS, et al. CYP2C9 and CYP2C19 genetic polymorphisms: frequencies in the south Indian population. Fundam Clin Pharmacol. 2005; 19: $101 \mathrm{e} 105$.

[10] Scott SA, Sangkuhl K, Gardner EE, et al. Clinical Pharmacogenetics Implementation Consortium guidelines.

[11] A study on the impact of CYP2C19 genotype and platelet reactivity assay on patients undergoing PCI P.C. Rath, Sundar Chidambaram, Pallavi Rath et al. indian heart journal 67 (2015), 114- e121.

[12] Anderson K M, Califf R M, Stone G W. et al Long - term mortality benefit with abciximab in patients undergoing percutaneous coronary intervention. J Am Coll Cardiol 2001372059-2065.2065.

[13] P Généreux, G W Stone R A Harrington et al. Impact of Intraprocedural Stent Thrombosis During Percutaneous Coronary Intervention. JACC (Journal of the American College of Cardiology), 2014-02-25, Volume 63, Issue 7, Pages 619-629. 